\title{
Remodelling after an Infarct: Crosstalk between Life and Death
}

\author{
Roberto Ferrari $^{a} \quad$ M. Malagù ${ }^{b} \quad$ S. Biscaglia ${ }^{b} \quad$ A. Fucilib $^{b} \quad$ P. Rizzo ${ }^{b}$ \\ ${ }^{a}$ Department of Cardiology and LTTA Centre, University of Ferrara, Ferrara, and ${ }^{\mathrm{b}}$ Maria Cecilia Hospital, \\ GVM Care and Research, E.S. Health Science Foundation, Cotignola, Italy
}

For editorial comment see p. 67

\section{Key Words}

Apoptosis · Hypertrophy $\cdot$ Myocardial infarction $\cdot$ Notch pathway $\cdot$ Remodeling

\section{Introduction}

In the first part of this review ('Can we improve myocardial protection during ischaemic injury?'), which appeared last month in Cardiology [1], we described some aspects of ischaemic and reperfusion damage. We emphasized the role of early reperfusion to salvage the ischaemic, but still viable, myocardium. However, we also recognized that timely reperfusion is not always feasible, and late reperfusion, although successful, is not able to prevent myocyte necrosis, enabling a vicious circle of damage. This circle of complex events ultimately degrades the anatomy and function of remaining viable myocytes, causing further ventricular dysfunction. This process is called 'remodelling'. Although this term became instantly popular and enduring, it hardly'speaks for itself in providing a precise and unequivocal definition. Thus, the perception of the term 'cardiac remodelling' varies according to whether one is a cardiologist, assessing the effects of remodelling on the volume and pumping capac- ity of the ventricle, a pathologist, for whom remodelling means hypertrophy, fibrosis or apoptosis, or a molecular biologist, who considers remodelling in terms of up- or down-regulation of various pathways and proteins in myocytes and the interstitium with a typical embryonic phenotype.

As is often the case in cardiology, a term may be used in an attempt to encapsulate a highly complex concept with a single, intriguing and occasionally cryptic name. Remodelling seems to be a prototypical example of this phenomenon and is probably the reason why we do not have a universal definition for remodelling, arguing even whether it is more appropriate to consider the term 'cardiac' versus 'myocardial' remodelling [2]. The truth is that remodelling is an evolving concept, originally applied almost exclusively to changes in cardiac structure occurring to the left ventricle (LV) after myocardial infarction, but recently also applied to the right ventricle and the atria $[3,4]$. It was also quickly recognized that the changes that characterize remodelling after infarction could have other causes, i.e. hypertension, aortic valve stenosis, myocarditis, valvular regurgitation and other forms of myocardial injury. Equally, it has become evident that remodelling occurs also in healthy subjects. Every heart experiences remodelling during growth from

\section{KARGER}

E-Mail karger@karger.com

www.karger.com/crd
(C) 2016 S. Karger AG, Basel

0008-6312/16/1352-0068\$39.50/0
Roberto Ferrari, MD, Chair of Cardiology

Ospedale di Cona

Azienda Ospedaliero-Universitaria di Ferrara

Via Aldo Moro 8, IT-44124 Cona (Italy)

E-Mail fri@unife.it 
childhood to adulthood and old age, reflecting myocyte proliferation and increasing in volume [5]. Pregnancy also causes temporary remodelling just in a few months [5]. Exercise training effects remodelling of the heart within few weeks or months; in some people, these changes may have pathological consequences [6]. It is, therefore, not surprising that there is the need to distinguish between 'physiological' and 'pathological' remodelling. Pathological remodelling is not limited to the ventricles, as the atria can undergo remodelling (a major substrate for atrial fibrillation). Even the vasculature can remodel, with clear implications for hypertension. The concept of cardiac remodelling is also pivotal in explaining the beneficial effects of many drugs on the outcome of heart failure (HF), such as angiotensin-converting enzyme inhibitors (ACEI), angiotensin II receptor blockers (ARB), $\beta$-blockers, mineralocorticoid receptor antagonists (MRA) or ivabradine, for example [2]. Not only can pharmacological interventions reshape the ventricle, but also devices such as biventricular pacemakers for cardiac resynchronization therapy, and have an anti-remodelling effect [2]. As always, the cardiologist sees only the tip of the iceberg, which is the improved cardiac structure and function, as consequences of these therapies. Under the surface, however, enormous changes in the heart's cellular and molecular structure must occur. If the molecular secrets of remodelling could be unlocked, more specific interventions to induce full cardiac repair and recovery might result. The scientific literature provides many reviews describing the molecular changes involved in remodelling, with some recently published [7-12]. This, however, is not the scope of our article. Instead, we propose a rather different approach to explain remodelling after infarction. This view was presented at the opening ceremony of the 11th International Congress of Cardiac Artery Disease (ICCAD) in Florence in November 2015.

\section{Remodelling after Myocardial Infarction}

In the first part of this review in Cardiology, we elaborated the concept that early interventions to open occluded coronary arteries may limit infarct size and subsequent remodelling, but late opening of coronary occlusions may do more harm than good [1]. Although myocardial infarction in humans is a complex process, it usually evolves areas of acute myocardial necrosis and adjacent regions, where viable and/or stunned myocytes exist. It is from these areas of necrosis that the process of ventricular remodelling rapidly begins within hours from the acute coronary occlusion and continues for almost a week [13]. The process involves cardiomyocyte lengthening and thinning of the infarct zone followed by inflammation and immune cell activation. Numerous cell types are involved in orchestrating this complex pathological response, as the heart consists of a heterogeneous population of cells, including cardiomyocytes, interstitial cells, connective tissue cells, smooth muscle cells, endothelial cells and other non-cardiomyocytes [9]. As a result, there is inflammatory and immunological activation, and the initiation of a repairing action, which will eventually cause re-absorption of necrotic tissue and stimulate production of interstitial collagen, leading to scar formation [14]. This early phase of remodelling occurs at the site of the infarct and may be considered beneficial. The myocyte becomes elongated as part of an initial compensatory process to maintain stroke volume after the loss of contractile tissue, and the scar prevents heart rupture (fig. 1). However, several studies of global LV chamber volumes and muscle mass show that early dilatation or, better, early remodelling might continue progressively, especially in elderly patients or in those with a large anterior wall infarct. This late phase of remodelling ( $\geq 1$ month after the infarct) occurs at a site distant from the infarcted area and involves myocytes which are still viable (fig. 1). These undergo complex and often opposite transformation, such as hypertrophy and/or apoptosis with a consistent common feature: a switch forward to the classical embryonic phenotype [15]. As a result, the end-systolic and -diastolic volumes increase progressively and the ejection fraction declines. These are important predictors of outcomes. Remodelling thus could be seen as a bridge between LV dysfunction and HF [16].

This second phase of remodelling does not necessarily occur after every infarct. Also, clinical studies show that it is not always progressive, as in the conventional picture of the so-called 'cardiovascular continuum', implying that once the LV dilatation starts, it inexorably leads to further worsening of cardiac function, $\mathrm{HF}$ and, ultimately, death, either by triggering arrhythmias or by end-stage congestion and pump failure [1]. This may have been true in the past, but, likely due to the benefits of modern therapy, is no longer the case for every patient. Definitively, the progression of remodelling can be stopped or delayed, whilst it is still controversial whether remodelling can actually be reversed. The most promising anti-remodelling strategy seems to be pharmacological therapy with agents that lower systolic and diastolic LV pressures, such as ACEI and MRA $[17,18]$, or agents that reduce heart rate, like $\beta$-blockers and ivabradine (fig. $2[19,20]$ ). These 
Fig. 1. LV remodelling: gross and microscopic architecture.

Fig. 2. Therapeutic intervention in pathological ventricular remodelling. CRT = Cardiac resynchronization therapy; ICD = implantable cardioverter-defibrillator; RAAS = renin-angiotensin-aldosterone system.
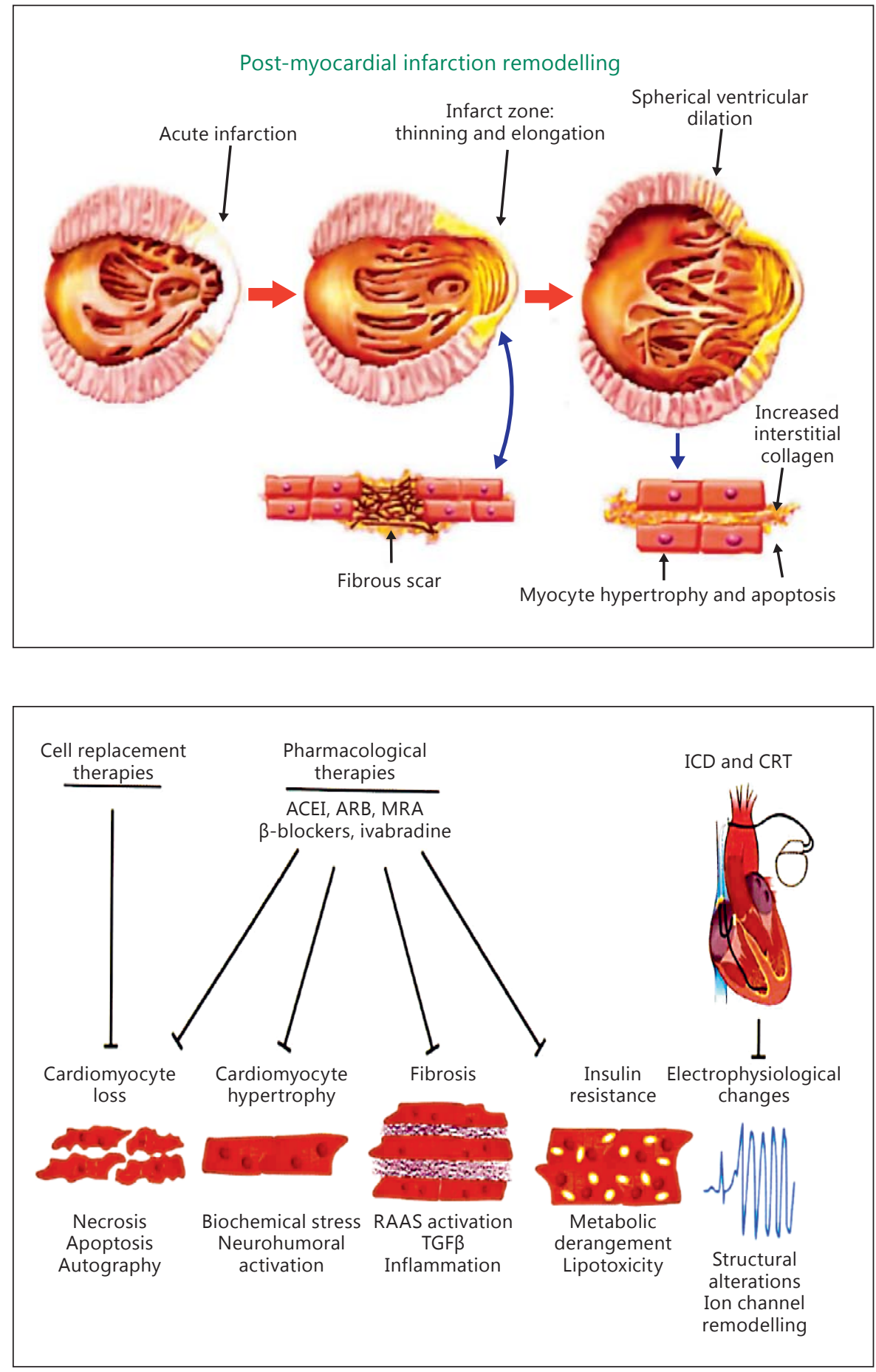

agents improve survival and counteract in different ways the transformation of viable functional myocytes into 'remodelled' dysfunctional myocytes. It is possible that the actual changes occurring in the remote myocardium are the major determinants of prognosis rather than the ven- tricular dilatation itself. The net effect of this early process is usually an acute increase in LV volume and a reduction in contractility, followed by recovery to a variable extent after a few days or weeks due to the resolution of stunning. 
Molecular Changes of Late Remodelling at a Remote Site

The molecular mechanisms involved in late remodelling are unclear. What is known is that when the heart remodels, its geometry changes: it becomes less elliptical and more spherical. There are also changes in the ventricular mass, composition and volume, all of which further adversely affect cardiovascular function [13]. At the cellular level, the late phase of remodelling encompasses changes including myocyte hypertrophy, apoptosis, increased fibrillar collagen, fibroblast proliferation and, eventually fibrosis. These changes are not exclusive for myocytes. Vascular smooth muscle cells, endothelial cells and leukocytes are equally involved in the remodelling process by promoting fibrosis, vascular stiffness, endothelial dysfunction, deregulated angiogenesis and inflammation. Recent papers have comprehensively reviewed the intercellular and intracellular signalling networks implicated in late cardiac remodelling [21-23]. Here, we focus on a particular feature of the changes described in the remodelled tissue, mainly the genomic shift toward a typical embryonic genome expression and on the Notch signalling pathways, as this system may be involved in the relationship between hypertrophy and apoptosis [24].

The finding of both apoptotic and hypertrophied myocytes in the remodelled myocardium is surprising as apoptosis is a clear expression of death, whereas hypertrophy could be considered expression of life, particularly in the context of the adult heart, which normally comprises terminal cells that do not easily reproduce. Relevant to our discussion is the recognition that in embryonic life, myocytes undergo the life-and-death cycle: they die from apoptosis and do regenerate. It follows that the presence of these two opposite signals in the remodelled zone indicates the re-instatement of the life/death cycle as in embryonic myocytes [15]. This is also confirmed from cardiac biopsies of patients with HF undergoing transplantation or from experimental animals showing that remodelled myocytes exhibit the embryonic phenotype with embryonic myofilaments, sarcoplasmic reticulum ATPase and granuli of atrial natriuretic peptide in the ventricle as well as ventricular presence of if channels and expression of if current, typical markers of foetal myocytes [15].

This is probably a stereotypical cellular response to a biochemical, anatomical and functional stress induced by the infarct. Although hypertrophy and apoptosis represent different phenotypic outcomes, the intracellular signals leading to one of the two conditions are multiple;
Notch signalling is likely to be involved and to play an important role, as it is the ultimate determinant of the cell fate [24]. Whichever the ultimate determinant, an important question is how a stressor that originates from the infarct site spreads to the remote, still viable myocytes.

In the course of an infarct, myocytes die from necrosis, a very different form of death from apoptosis. Contrary to apoptosis (which could be considered a physiological type of death), necrosis is not programmed by the nuclei. It occurs in response to the acute ischaemia which, in turn, depends on thrombotic occlusion of the coronary artery subtending the infarct area. Necrotic death involves millions of myocytes at once, leading to an immunological reaction, whereas apoptosis involves a single cell at a time without immunological activation. Furthermore, the physical result of this typology of deaths is different. In apoptotic death, the cell itself dissolves into several (millions!) small apoptotic bodies which are neutralized by macrophages, whilst myocytes dying from necrosis undergo drastic geometrical changes in their shape: from rectangular and elongated, they become spherical and significantly smaller. The necrotic myocytes shrink as a consequence of the unexpected ischaemia-induced dissolution of intracellular organelles such as mitochondria, sarcoplasmic reticulum and nuclei with subsequent release of their calcium into the cytosol resulting in hypercontraction of the myofilaments (fig. 3). The clinical counterpart of these molecular changes is the increase in diastolic ventricular pressure and the reduced compliance during the acute and later phase of the infarct. Such drastic mechanical changes result in stretching of the sarcolemma of the adjacent still viable myocytes at the level of the intercalated disc, as schematically shown in figure 3. Such mechanical stretching, in turn, in an autocrine manner, activates signalling systems which directly, or indirectly, determine a switch toward the foetal genetic programme at nuclear level [15]. The Notch pathway is a system of communication between adjacent cells and is likely, even though not exclusively, involved [24]. It follows that, after the infarct, at a molecular level, a clear mechanical/molecular coupling occurs with the myocytes which, in turn, act as biochemical sensors $[25,26]$. A possible series of events could be: the dying myocyte, as a result of the acute ischaemia, generates a mechanical stretch which is transmitted via the sarcolemma to the remote, still viable myocytes. The stretch itself is coupled with a series of biochemical/molecular alterations with the release of hormones and cytokines, and the activation of several different pathways determining, at a nuclear 


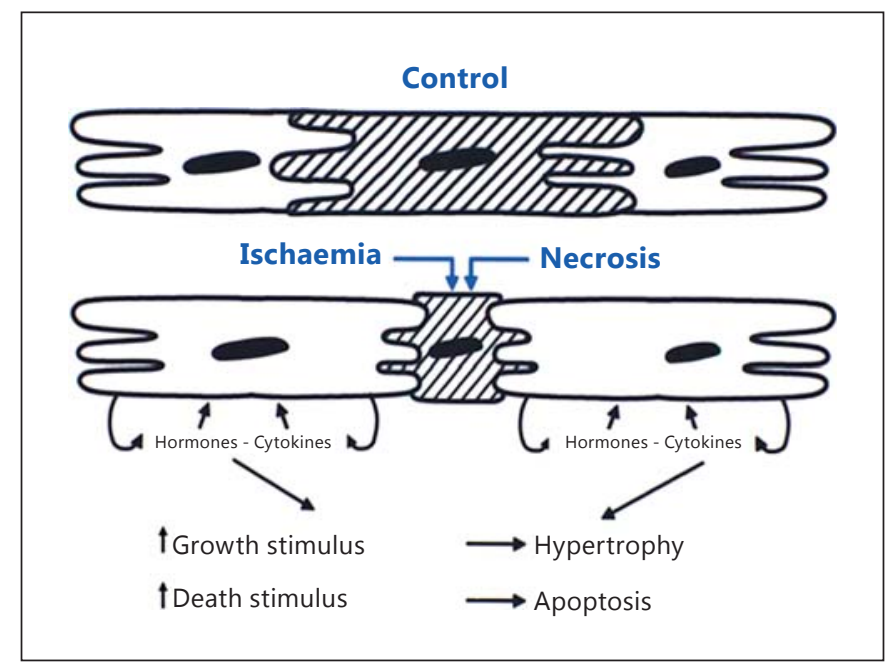

Fig. 3. Death and survival paths have strong similarities. Although hypertrophy and apoptosis represent distinct phenotypic outcomes, the intracellular signals are very similar, involving the renin-angiotensin system, protein kinase paths and components of MAP kinase (p38 $\beta / \alpha$ and Jun-N-terminal kinase), which have terminal signals that lead either to hypertrophy or survival.

level, a switch toward the foetal genetic programme. Gradually, the contractile proteins and the system deputed to $\mathrm{Ca}^{2+}$ homeostasis start to resemble those of the embryonic myocytes resulting in a delay of the speed of contraction as well as in a reduction in the force of contraction. In addition, the so-called life/death cycle is reinstated leading to hypertrophy and apoptosis of the adult still viable myocytes distant from the infarct. Complete myocyte regeneration is not possible, leading just to hypertrophy, whilst apoptosis can fully occur. In the early phases of this sequence of events, likely there is a matching between apoptosis and hypertrophy but, when apoptosis prevails, there will be a progressive loss of myocytes with fibrotic substitution. The hypertrophied myocytes themselves are dysfunctional, and the remaining ones show embryonic features with reduced contractile properties. As a result, the ventricle further enlarges, changes its shape, the ejection fraction is reduced, and symptoms of HF progress.

Stretching of the sarcolemma, however, is not the only cause for re-instatement of the embryonic phenotype. Several other extracellular components are likely to play an important role. Judged from the documented capacity of ACEI, $\beta$-blockers, MRA and ivabradine to attenuate the remodelling process, it is likely that angiotensin II, noradrenaline, aldosterone and heart rate also promote the molecular/genomic changes in remodelling. As

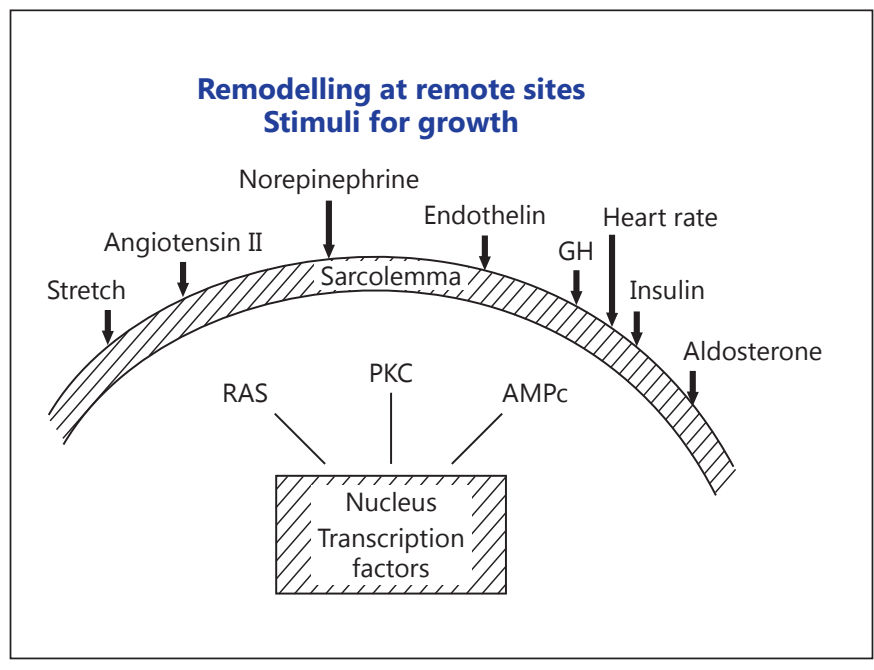

Fig. 4. Nuclear changes leading to the embryonic programme depend on signal systems such as those activated by the renin-angiotensin system (RAS), protein kinase $\mathrm{C}$ (PKC) and cyclic AMP $(\mathrm{AMPc})$. Extracellular signals include angiotensin II, noradrenaline and aldosterone, as well as stretch.

shown in figure 4 , it is relevant to underline that all these substances acting outside the cell membrane modulate a variety of intracellular signalling networks, such as protein kinases (AKT), Notch and intracellular RAS, just to mention some, all of which may be involved in remodelling $[27,28]$.

\section{Regulatory Processes: Learning from Oncology}

Whatever the induction, either mechanical (stretch and heart rate changes) or chemical (angiotensin II, noradrenaline and aldosterone), the above-mentioned steps are likely to be closely regulated to many different systems which, under physiological conditions, prevent these series of events from occurring. For example, the adult heart develops different survival pathways which inhibit myocyte apoptosis. Apart from the role of Notch, which is examined in the next paragraph, cardiotrophin-1, a gp130 receptor-dependent cytokine, is a potent inhibitor of apoptosis [29] and it has been shown to provide cardiac protection against ischaemia in experimental animals [26]. Mice with abolition of cardiotrophin-1 and with dysfunction of gp 130 receptors display massive myocyte apoptosis and high mortality in response to just moderate hypertension [30]. Neuregulin also suppresses apoptosis, and ablation of neuregulin or of its receptors, 
$\mathrm{ERB}_{2}$ and $\mathrm{ERB}_{4}$, linked to gp185, leads to $\mathrm{HF}$ in mice [31]. On the other hand, CT-1, a new member of the interleukin- 6 cytokine family, activates myocyte hypertrophy, with embryonic gene expression and organization [32]. After an infarct, there is an increase in the levels of the interleukin- 6 cytokine family with consequent activation of gp130 and $\mathrm{ERB}_{2}$ linked to gp185 receptors, which favours adaptive hypertrophy and inhibits apoptosis, thus exerting a beneficial effect. Conversely, already 3 days after an infarct, mice with gp 130 ventricular knockout display rapid onset of dilated cardiomyopathy characterized by an embryonic phenotype with massive apoptosis involving over $30 \%$ of the ventricular myocytes $[15,31,32]$.

Further evidence of the important protective role of gp130 comes from patients with breast cancer undergoing treatment with trastuzumab $[33,34]$, which is an antibody able to block gp130-gp185 and is used as an adjuvant therapy for gp130 and $\mathrm{ErbB}_{2}$-positive breast cancer. By blocking these receptors, trastuzumab re-instates the intracellular death signals and apoptosis of cancer cells and, at the same time, it reduces the life signals slowing down cancer growth. Randomized clinical trials have shown that trastuzumab has positive effects on cancer progression but was unexpectedly associated with an increased incidence of HF due to dilated cardiomyopathy with embryonic phenotype, particularly when given concomitant with or after anthracycline treatment $[33,34]$. It is likely that the effect of blocking gp130 by trastuzumab occurs also in myocytes, thus re-instating apoptosis. As a consequence, in the heart, the death signals prevail over the survival signals; the ventricle undergoes remodelling and eventually HF occurs [15]. This is a unique piece of translational research in which oncologists unexpectedly proved the importance of gp130 and gp185 in cardiac remodelling.

\section{Crosstalk between Notch Signalling and Other Pathways Involved in Remodelling}

Notch signalling is rather complex. Here we attempted to provide a single explanation of it. Humans have four Notch receptors (Notch 1-Notch4) and five ligands [delta-like (Dll) 1, Dll3 and Dll4, Jagged 1 and Jagged2] located on the cell surface of neighbouring cells. Figure 5 shows the interaction between Notch receptors and their ligands. Binding of a ligand to the receptor triggers two proteolytic cleavages, leading to the release of the Notch intracellular domain (NIC), which is the active form of
Notch. NIC enters the nucleus, binds to the RBP-Jk transcription factor (recombination signal binding protein for immunoglobulin $\kappa J$ region) and regulates the transcription of target genes. The Notch pathway plays a major role during heart development when physiological remodelling also occurs [35]. Accordingly, mutations in components of the Notch signalling pathway are linked to congenital abnormalities, such as bicuspid aortic valve disease, calcification of the heart valve and LV non-compaction cardiomyopathy $[36,37]$.

Recently, Notch dysregulation has also been linked to HF progression and mortality [38]. Also, the anti-cancer agent demcizumab, an antibody against the Notch ligand DII4, which interferes in tumour angiogenesis, is associated with the risk of HF when administered over a prolonged interval [39]. In the adult rat myocardium, Notch signalling is absent under normal physiological conditions, but it becomes transiently reactivated after myocardial infarction in the border zones [40]. Expression of Notch signalling components is present in cardiac biopsies from HF patients [41]. These observations suggest a role for Notch signalling in the repair of ischaemic or overloaded myocardium. This hypothesis is supported by studies in animal models, in which inhibition of Notch signalling increases the size of the infarct scar [42], and activation of Notch1 signalling in cardiomyocytes results in reduced myocardial damage following an ischaemic insult [43]. Also, activation of Notch signalling has been identified as one of the molecular mechanisms by which pre- and post-ischaemic conditioning reduce ischaemiareperfusion damage [44]. Treatment with high-mobility group box chromosomal protein (HMGB1), a nuclear protein that acts as a cytokine when released by necrotic and inflammatory cells, promotes heart repair by activating Notch [45].

The Notch network could be involved both in the early and late phases of remodelling. In early remodelling, a delicate equilibrium exists between the proinflammatory response, which is needed to clear the debris caused by extensive myocyte death, and the following fibrotic response, which fills the empty space created by necrotic tissue and generates the scar (fig. 1). For effective healing, the two responses need to be carefully orchestrated. Notch is an important modulator of inflammation, and the use of Notch inhibitors reduces the inflammatory response in different animal models. At the same time Notch crosstalk with the transforming growth factor- $\beta$ (TGF $\beta$ ) in cardiomyocytes promotes fibrosis by enhancing the transition of myoblasts to fibroblasts and inhibits inflammation [46]. In addition, Notch signalling promotes myocardial 


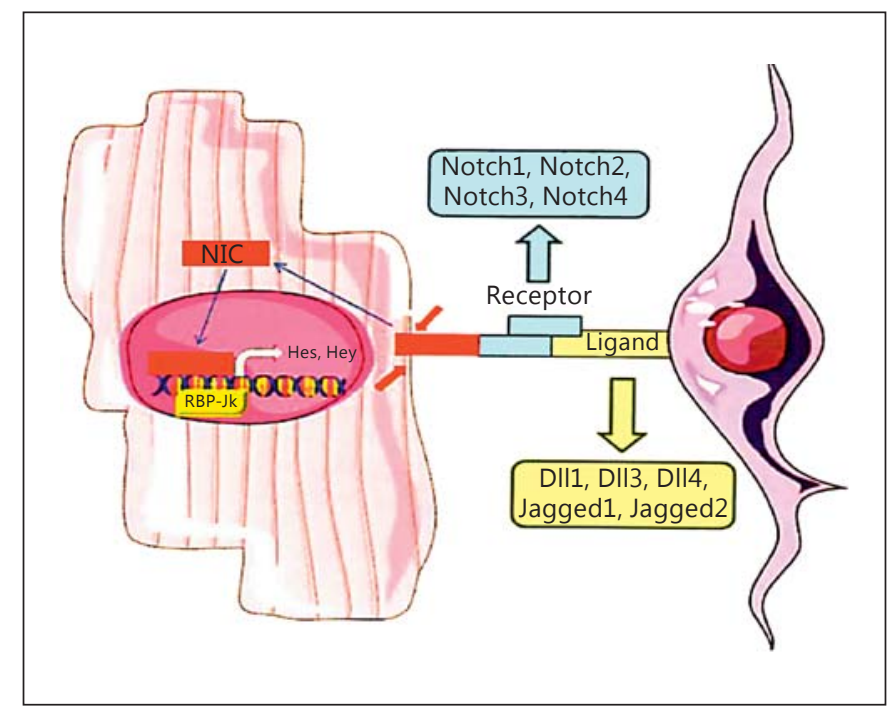

Fig. 5. Interaction between Notch receptors and their ligands. The interaction between Notch receptors (Notch1-Notch4) and their ligands (Jagged1, Jagged 2, Dll1, Dll3 and Dll4) induces cleavage of the receptors by two proteases (red arrows), which release NIC. In the nucleus, NIC binds to the transcription factor, RBP-Jk and activates transcription of target genes (Dll and RBP-Jk). Hes = Hairy and enhancer of split; Hey = hairy/enhancer-of-split related with the YRPW motif.

angiogenesis, activating the Notch ligand DII4 [27]. Other important effects of Notch on the vasculature are related to its effect on the endothelium. Notch I activation is linked to inhibition of the expression of miR-155, a miRNA associated with eNOS malfunction. In this setting, Notch exerts a key role in repairing the necrotic myocytes and the damaged endothelium [27].

In the late phase of remodelling, Notch is likely to play a role in the delicate regulation of the life/death cycle or, in other words, the balance of the apoptotic death and the hypertrophic response of the viable cardiomyocyte, which is the hallmark of the progression from infarct to HF.

The important role played by Notch in the protection of cardiomyocyte survival has been reported by several authors. A study on cardiac remodelling secondary to haemodynamic overload due to hypertension has shown that activation of Notch I signalling in cardiomyocytes limits the hypertrophic response, whilst treatment with Notch inhibitors leads to exacerbated cardiac hypertrophy, altered function, and increased mortality. In cardiomyocytes grown under hypoxic conditions, Notch I activation leads to increased expression of anti-apoptotic genes, whereas pharmacological inhibition of Notch signalling causes increased apoptosis [43]. In a rat model of

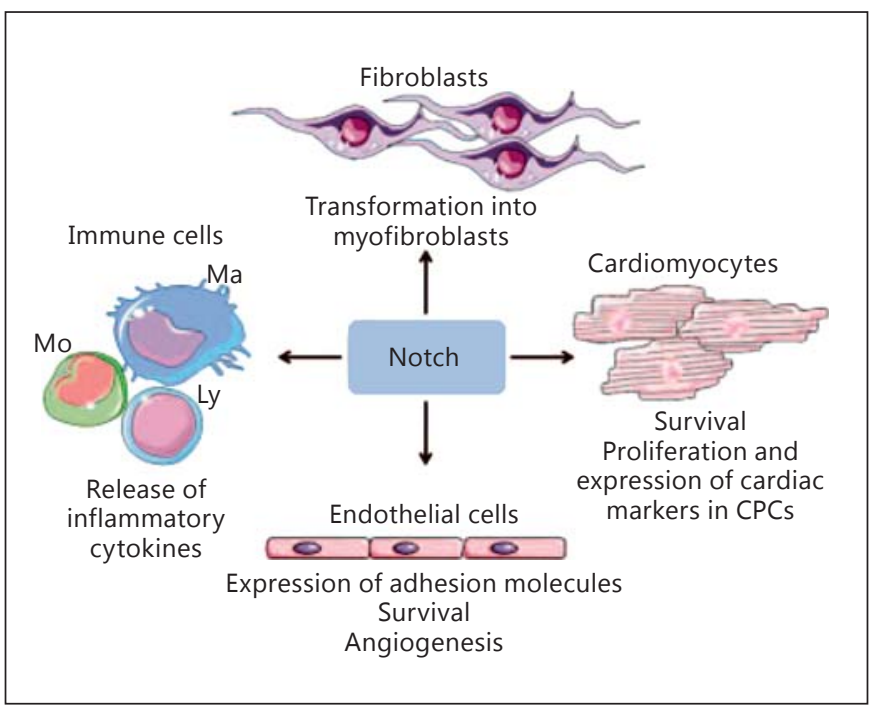

Fig. 6. Notch signalling as a therapeutic target for LV remodelling. By mediating the interactions between adjacent cells, Notch controls fibrous tissue deposition, cardiac hypertrophy, angiogenesis and inflammation; therefore, it could represent a therapeutic target for the reduction in LV remodelling. This figure summarizes the cellular functions modulated by Notch signalling in the myocardium. $\mathrm{CPC}=$ Cardiac progenitor cells; $\mathrm{Ly}=$ leukocytes; $\mathrm{Ma}=$ macrophages; $\mathrm{Mo}=$ monocytes.

myocardial infarction, Notch I is reactivated in cardiomyocytes near the border zone, leading to activation of the pro-survival Akt pathway. Similarly, in mice genetically modified to express the active form of Notch I in cardiomyocytes, a decreased number of apoptotic cells following myocardial infarction has been found compared with mice without the modification [43]. Recent work has shown that a reduction in cardiomyocyte apoptosis linked to notch activation is part of the molecular mechanism by which ischaemic pre- and post-conditioning exert their cardioprotective activity in the infarcted heart of the rat. Both during apoptosis and hypertrophy, the activation of Notch in cardiomyocytes is mediated by Jagged1, which is present on the surface of adjacent cardiomyocytes and results in reducing signals of death (apoptosis) and increasing those of life (hypertrophy) [27].

Figure 6 summarizes the possible role of Notch signalling in the early and late phases of remodelling. It is important to further advance our knowledge of the Notch pathway after an infarct. Following the disappointing results shown by clinical trials that failed to improve cardiac function by intracoronary injection of stem cells, the hope now relies on the in vivo delivery of cytokines, growth factors or modulators of systems, such as the 
Notch pathway, able to suppress the death signals and to enhance the possible regenerative potential of the heart, even if limited to hypertrophy. Our colleagues working on cancer have followed this route with success - cardiology may learn from them.

\section{Final Remarks}

As stated in the first part of our review [1], currently, early reperfusion is the only viable approach to enable cardiac recovery and abolish or, at least, reduce myocardial damage. If not successful, reperfusion might cause further damage, which eventually will give rise to complex molecular alterations leading to HF following a process of remodelling. Remodelling after myocardial infarction is clinically relevant and will increase in relevance with the increasing age of the population. Symptoms and the changes of ventricular shape need not be the exclusive guide as to when therapy should be initiated, and the choice of therapy should take into account all the underlying components that contribute to remodelling. The available molecular explanations for the process suggest that therapy should be implemented as early as possible. The next steps will be the determination of how the information generated from cellular and molecular models can be used, together with data from clinical trials, to ensure optimal therapy at an appropriate time. ACEI, $\beta$-blockers, anti-aldosterone drugs, ivabradine and cardiac resynchronization therapy, which reduce morbidity and mortality and improve a number of remodelling parameters, currently offer the best therapeutic approach, but this is not enough. The challenge is to develop new and more specific treatments that may be even more effective in reversing the structural LV abnormalities. Of course, all of this is of paramount importance as HF due to ischaemic heart disease is one of the leading causes of death worldwide. However, once again, the best therapy for remodelling will be to avoid its occurrence simply by early reperfusion.

\section{Acknowledgments}

This work was supported by a grant from Fondazione Anna Maria Sechi per il Cuore (FASC), Italy. The funders had no role in the preparation and decision to publish the paper.

\section{Conflict of Interest}

Roberto Ferrari reports that he received an honorarium from Servier for steering committee membership, consulting and speaking, as well as support from Servier for travel to study meetings. In addition, he received personal fees from Amgen, Boehringer-Ingelheim, Novartis, Merck Serono and Irbtech.

\section{References}

1 Ferrari R, Biscaglia S, Malagù M, Bertini M, Campo G: Can we improve myocardial protection during ischaemic injury? Cardiology 2016;135:14-26.

2 John G, Cleland F: Cardiac remodelling: what has changed over the past 10 years? Dialogues Cardiovasc Med 2015, vol 20.

3 Pellicori P, Zhang J, Lukaschuk E, et al: Left atrial function measured by cardiac magnetic resonance imaging in patients with heart failure: clinical associations and prognostic value. Eur Heart J 2015;36:733-742.

4 Bourantas C, Loh PH, Bragadeesh T, et al: Relationship between right ventricular volumes measured by cardiac magnetic resonance imaging and prognosis in patients with chronic heart failure. Eur J Heart Fail 2011;13:52-60.

5 Nikitin NP, de Silva R, Witte KK, et al: Normal age- and sex-related values of left ventricular volumes, myocardial mass and ejection fraction obtained with cardiac magnetic resonance using FIESTA imaging. J Cardiovasc Magn Reson 2004;6:163-164.
6 Weiner RB, Wang F, Berkstresser B, et al: Regression of 'gray zone' exercise-induced concentric left ventricular hypertrophy during prescribed detraining. J Am Coll Cardiol 2012;59:1992-1994.

7 Hermans KC, Daskalopulos EP, Blankesteijn WM: The Janus face of myofibroblasts in the remodelling heart. J Mol Cell Cardiol 2016;91: $35-41$.

8 Taylor AJ, Chain W: Post-infarction LV remodelling: remote changes do not necessarily occur remotely from time of infarction. JACC Cardiovasc Imaging 2015;8:790-792.

9 Frieler RA, Mortensen RM: Immune cell and other noncardiomyocyte regulation of cardiac hypertrophy and remodelling. Circulation 2015;131:1019-1030.

10 Spaich S, Katus HA, Backs J: Ongoing controversies surrounding cardiac remodelling: is it black and white - or rather fifty shades of gray? Front Physiol 2015;6:202.
11 Lindsey ML, Iyer RP, Jung M, DeLeon-Pennell KY, Ma Y: Matrix metalloproteinases as input and output signals for post-myocardial infarction remodelling. J Mol Cell Cardiol 2016; 91:134-140.

12 D'Elia N, D'hooge J, Marwick TH: Association between myocardial mechanics and ischaemic LV remodelling. JACC Cardiovasc Imaging 2015;8:1430-1443.

13 Burchfield JS, Xie M, Hill JA: Pathological ventricular remodeling: mechanisms: part 1 of 2. Circulation 2013;128:388-400.

14 Anversa P, Olivetti G, Capasso JM: Cellular basis of ventricular remodeling. JACC 2000; 35:569-582.

15 Ferrari R, Ceconi C, Campo G, Cangiano E, Cavazza C, Secchiero P, Tavazzi L: Mechanisms of remodelling: a question of life (stem cell production) and death (myocyte apoptosis). Circ J 2009;73:1973-1982.

16 Pellicori P, Carubelli V, Zhang J, et al: IVC diameter in patients with chronic heart failure: relationships and prognostic significance. JACC Cardiovasc Imaging 2013;6:16-28. 
17 Abdulla J, Barlera S, Latini R, et al: A systematic review: effect of angiotensin converting enzyme inhibition on left ventricular volumes and ejection fraction in patients with a myocardial infarction and in patients with left ventricular dysfunction. Eur J Heart Fail 2007; 9:129-135.

18 Weir RA, Mark PB, Petrie CJ, et al: Left ventricular remodeling after acute myocardial infarction: does eplerenone have an effect? Am Heart J 2009;157:1088-1096.

19 Freemantle N, Cleland J, Young P, Mason J, Harrison J: $\beta$ blockade after myocardial infarction: systematic review and meta regression analysis. BMJ 1999;318:1730-1737.

20 Böhm M, Swedberg K, Komajda M, Borer JS, Ford I, Dubost-Brama A, Lerebours G, Tavazzi L; SHIFT Investigators: Heart rate as a risk factor in chronic heart failure (SHIFT): the association between heart rate and outcomes in a randomised placebo-controlled trial. Lancet 2010;376:886-894.

21 Xie M, Burchfield JS, Hill JA: Pathological ventricular remodeling: therapies: part 2 of 2 . Circulation 2013;128:1021-1030.

22 Frangogiannis NG: The inflammatory response in myocardial injury, repair, and remodeling. Nat Rev Cardiol 2014;11:255-265.

23 Burchfield JS, Xie M, Hill JA: Pathological ventricular remodeling: mechanisms: part 1 of 2. Circulation 2013;128:388-400.

24 Guruharsha KG, Kankel MW, ArtavanisTsakonas S: The Notch signaling system: recent insights into the complexity of a conserved pathway. Nat Rev Genet 2012;13:654666.

25 Chien K: Genomic circuits and the interactive biology of cardiac diseases. Nature 2000;407: 227-232.

26 Chien KR: Stress pathways and heart failure. Cell 1999;98:555-558.

27 Rizzo P, Ameri P: What role does intracellular and intercellular signaling play in cardiac remodeling? Dialogues Cardiovasc Med 2015 vol 20.
28 Rizzo P, Miele L, Ferrari R: The Notch pathway: a crossroad between the life and death of the endothelium. Eur Heart J 2013;34:25042509.

29 Taga T, Kishimoto T: gp130 and the interleukin-6 family of cytokines. Annu Rev Immunol 1997;15:797-819.

30 Wang Y, Huang S, Sah VP, Ross J Jr, Brown $\mathrm{JH}, \mathrm{Han} J$, et al: Cardiac muscle cell hypertrophy and apoptosis induced by distinct members of the $\mathrm{p} 38$ mitogen-activated protein kinase family. J Biol Chem 1998;273:21612168.

31 Zhao YY, Sawyer DR, Baliga RR, Opel DJ, Han X, Marchionni MA, et al: Neuregulins promote survival and growth of cardiac myocytes: persistence of ErbB2 and ErbB4 expression in neonatal and adult ventricular myocytes. J Biol Chem 1998;273:10261-10269.

32 Ferrari R: Healthy versus sick myocyte: metabolism, structure, and function. Defining the friends and foes. Dialogues Cardiovasc Med 2009;14:227-243.

33 Perik PJ, de Korte MA, van Veldhuisen DJ, Gietema JA, Sleijfer DT, de Vries EG: Cardiotoxicity associated with the use of trastuzumab in breast cancer patients. Expert Rev Anticancer Ther 2007;7:1763-1771.

34 Ewer MS, Lippman SM: Type II chemotherapy-related cardiac dysfunction: time to recognize a new entity. J Clin Oncol 2006;23:29002902.

35 De la Pompa JL, Epstein JA: Coordinating tissue interactions: Notch signaling in cardiac development and disease. Dev Cell 2012;22: 244-254.

36 High FA, Epstein JA: The multifaceted role of Notch in cardiac development and disease. Nat Rev Genet 2008;9:49-61.
37 Luxan G, Casanova JC, MartinezPoveda B, et al: Mutations in the NOTCH pathway regulator MIB1 cause left ventricular noncompaction cardiomyopathy. Nat Med 2013;19:193201

38 Abraityte A, Gullestad L, Askevold ET, et al: The Notch ligand Delta-like 1 is elevated and associated with mortality in patients with symptomatic aortic stenosis. Int J Cardiol 2014;180:18-20.

39 Smith DC, Eisenberg PD, Manikhas G, et al: A phase I dose escalation and expansion study of the anticancer stem cell agent demcizumab (anti-DLL4) in patients with previously treated solid tumors. Clin Cancer Res 2014;20: 6295-6303.

40 Gude NA, Emmanuel G, Wu W, et al: Activation of Notch-mediated protective signaling in the myocardium. Circ Res 2008;102:10251035.

41 Oie E, Sandberg WJ, Ahmed MS, et al: Activation of Notch signaling in cardiomyocytes during post-infarction remodeling. Scand Cardiovasc J 2010;44:359-366.

42 Boni A, Urbanek K, Nascimbene A, et al: Notch1 regulates the fate of cardiac progenitor cells. Proc Natl Acad Sci USA 2008;105: 15529-15534.

43 Kratsios P, Catela C, Salimova E, et al: Distinct roles for cell-autonomous Notch signaling in cardiomyocytes of the embryonic and adult heart. Circ Res 2010;106:559-572.

44 Zhou XL, Zhao Y, Fang YH, Xu QR, Liu JC Hes1 is upregulated by ischemic postconditioning and contributes to cardioprotection. Cell Biochem Funct 2014:32:730-736.

45 Limana F, Esposito G, Fasanaro P, et al: Transcriptional profiling of HMGB1-induced myocardial repair identifies a key role for Notch signaling. Mol Ther 2013;21:18411851

46 Frangogiannis NG: Targeting the transforming growth factor (TGF)-beta cascade in the remodeling heart: benefits and perils. J Mol Cell Cardiol 2014;76:169-171. 